\title{
Analysis And Implementation of Noekeon Algorithms For Encryption and Description of Text Data
}

\author{
${ }^{1}$ Noverta Efendi, ${ }^{2}$ Fauzan Azim \\ 1,2Electronic Engineering Vocational Education, Faculty of Teacher Training and Education, \\ Universitas Muhammadiyah Riau, Jalan Tuanku Tambusai, Kota Pekanbaru, Provinsi Riau. \\ Email : noverta04@gmail.com ${ }^{1}, \underline{\text { fauzanazim@gmail.com }}^{2}$
}

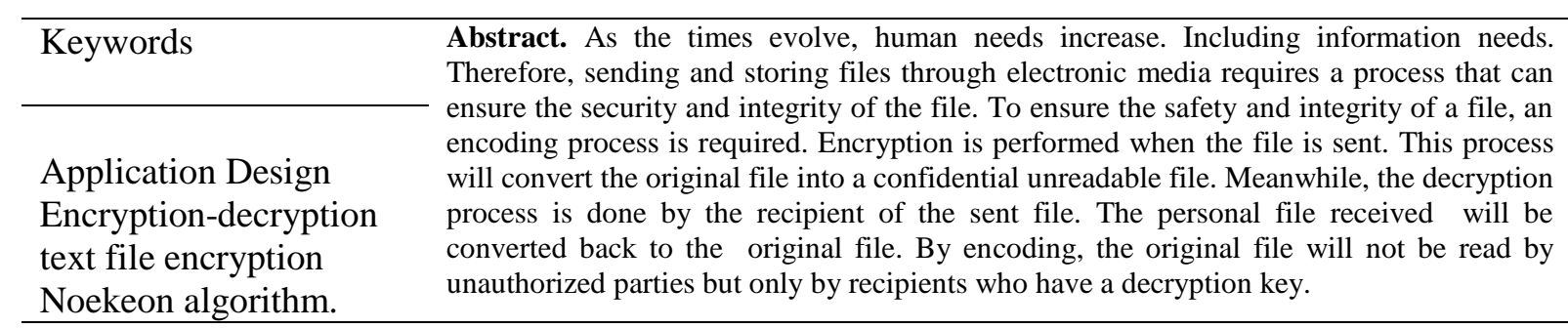

\section{INTRODUCTION}

Currently, one of the ways used for file security is to use a cryptographic system that is to encode the contents of the information (plaintext) into content that is not understood through the encryption process (chipper) and to regain the original data, the decryption process (decipher) accompanied by using the correct key[1]. But with the development of the science of encoding or cryptography, efforts to obtain the key can be made by anyone, including those who are not authorized to have such information. Therefore, researchers on cryptography will constantly evolve to get increasingly powerful cryptographic algorithms, making efforts to break down cryptographic codes unlawfully become more complex[2].

Information communication today has expanded widely. Information communication in exchanging messages and transactions until the exchange of confidential information has used computer technology and a vast and complex global network. The breadth of information communication utilizing computer technology and global networks today led to the emergence of parties who want to steal such information[3]. The vast and complex global network makes it almost impossible to protect messages right to their destination. The threat of information theft has led many researchers to develop techniques or methods to provide security to the content of information in notes, one of which is data encoding techniques or cryptography[4].

Cryptography is the science and art of maintaining the confidentiality of messages by encoding them into a form that is no longer understood. The purpose of cryptography is to provide security in the form of / privacy, data integrity, authentication, non-repudiation[5]. In the field of cryptography, there are two main processes, encryption, and decryption[6]. Encryption is the process of encoding plaintext or messages into ciphertext. Decryption is the process of returning the ciphertext to its original plaintext[7].

\section{METHOD}

The early stage is the determination of the use of materials related to the research conducted. At this stage will be done identifying the problem and selecting algorithms and methods to solve it. Issues are found by following current technology issues and developments and studying research that has been done and published through scientific journals. The problem to be researched is how to secure data. To avoid theft or leakage of data on cloud storage, the encryption system is implemented with noekeonalgorithm. Stages with the following flow: 


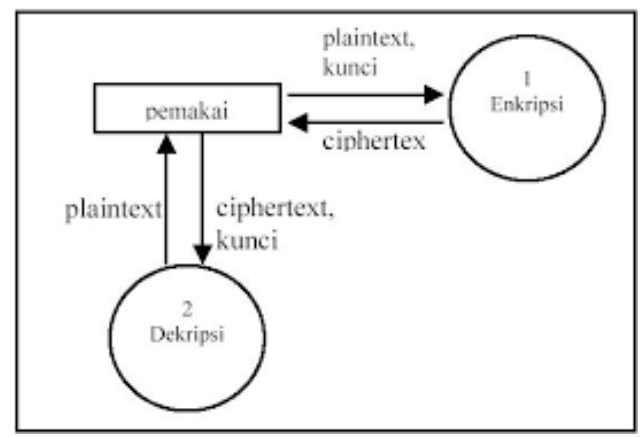

Figure 1. Noekeon Algorithm Work Process

\subsection{Noekeon}

Noekeon is a repeating code block with a block length and a key length of 128 bits each, consisting of a simple repeating spin transformation application, followed by an output transformation. Noekeon has 16 rounds (Nr) of iterations[8]. Each game is carried out three modifications, namely theta, offset shift consisting of two changes Pill and Pil2, and gamma[9].

Critical scheduling is done by converting a 128-bit primary key (code-key) into a working key. In Noekeon, there is a mode in which key scheduling is not performed, called direct-key mode, which serves the key scheduling process to eliminate related-key attack patterns[10].

\section{RESULT AND DISCUSSION}

The encryption and decryption process of Noekeon's algorithm uses the key generation or scheduling process used in each round of encryption and decryption. The key stages of the neocon algorithm are as follows:

1. Input the user key.

2. They are padding the user key if the key length is less than 16 characters.

3. Form A0-A3 blocks of 32 bits or four characters each.

4. Performing a key scheduling round of 16 rounds (i), the operations on each game are as follows:

1. Perform xor A0 operations with RC(i).

2. Perform inverse theta operations against A0-A3.

3. Perform rotate_left operations A $1<<1$

4. Perform operations rotate_left A2 $<<5$

5. Perform operations rotate_left A $3<<2$

6. Perform gamma operations against A0-A3.

7. Perform rotate_right operation $\mathrm{A} 1>>1$

8. Perform rotate_right operation $A 2>>5$

9. Perform operations rotate_right $\mathrm{A} 3>>2$

10.After the next round of operations, perform xor operations $\mathrm{A} 0$ and $\mathrm{RC}(16) .11 . \mathrm{A} 0-\mathrm{A} 3$ will be the DK(0)-DK(3) decryption key.

12. Perform inverse theta operations against A0-A3.

13. A0-A3 will be the key to $\operatorname{EK}(0)-\operatorname{EK}(3)$.

Table 1. RC Constants

\begin{tabular}{|c|c|c|}
\hline $\mathrm{i}$ & Formula & RC(i) \\
\hline 0 & - & $0 \times 80$ \\
\hline 1 & $\begin{array}{l}\mathrm{RC}[0] \text { And } 0 \mathrm{x} 80 !=0 \text { ? If True } \mathrm{Rc}[1]=\mathrm{Rc}[0] \ll<1 \text { Xor 0x1B If False RC[1]= Rc[0] } \\
1\end{array}$ & $0 x 1 a$ \\
\hline 2 & $\begin{array}{l}\mathrm{RC}[1] \text { And } 0 \mathrm{x} 80 !=0 \text { ? If True } \mathrm{Rc}[2]=\mathrm{Rc}[1]<<1 \text { Xor 0x1B If False RC[2]= Rc[1] }<< \\
1\end{array}$ & $0 \times 34$ \\
\hline 3 & $\begin{array}{l}\mathrm{RC}[2] \text { And } 0 \mathrm{x} 80 !=0 \text { ? If True } \mathrm{Rc}[3]=\mathrm{Rc}[2]<<1 \text { Xor 0x1B If False } \mathrm{RC}[3]=\mathrm{Rc}[2]<< \\
1\end{array}$ & $0 \times 68$ \\
\hline
\end{tabular}

Jurnal Info Sains : Informatikan dan Sains is licensed under a Creative Commons Attribution-Non Commercial 4.0 International License (CC BY-NC 4.0) 


\begin{tabular}{|c|c|c|}
\hline 4 & $\begin{array}{l}\mathrm{RC}[3] \text { And } 0 \times 80 \text { != } 0 \text { ? If True Rc[4] = Rc[3] }<<1 \text { Xor 0x1B If False RC[4]= Rc[3] }<< \\
1\end{array}$ & $0 x d 0$ \\
\hline 5 & $\begin{array}{l}\mathrm{RC}[4] \text { And } 0 \times 80 \text { != } 0 \text { ? If True Rc[5] = Rc[4] }<<1 \text { Xor 0x1B If False RC[5]= Rc[4] } \\
1\end{array}$ & 0xba \\
\hline 6 & $\begin{array}{l}\mathrm{RC}[5] \text { And } 0 \mathrm{x} 80 \text { != } 0 \text { ? If True Rc[6] = Rc[5] } \\
1\end{array}$ & $0 x 6 \mathrm{e}$ \\
\hline 7 & $\mathrm{RC}[6]$ And $0 \times 80 !=0$ ? If True $\mathrm{Rc}[7]=\mathrm{Rc}[6] \ll<1$ Xor 0x1B If False $\mathrm{RC}[7]=\mathrm{Rc}[6] \ll<1$ & $0 x d c$ \\
\hline 8 & $\begin{array}{l}\text { RC[7] And 0x80 != } 0 \text { ? If True Rc[8] = Rc[7] }<<1 \text { Xor 0x1B If False RC[8]= Rc[7] }<< \\
1\end{array}$ & 0xa2 \\
\hline 9 & $\begin{array}{l}\mathrm{RC}[8] \text { And } 0 \times 80 !=0 \text { ? If True } \mathrm{Rc}[9]=\mathrm{Rc}[8] \ll<1 \text { Xor 0x1B If False RC[9]= Rc[8] } \\
1\end{array}$ & $0 \times 5 \mathrm{e}$ \\
\hline 10 & $\begin{array}{l}\mathrm{RC}[9] \text { And } 0 \times 80 !=0 \text { ? If True } \mathrm{Rc}[10]=\mathrm{Rc}[9]<<1 \text { Xor 0x1B If False RC[10]= Rc[9] } \\
<<1\end{array}$ & $0 x b c$ \\
\hline 11 & $\begin{array}{l}\mathrm{RC}[10] \text { And } 0 \times 80 !=0 \text { ? If True } \mathrm{Rc}[11]=\mathrm{Rc}[10] \ll<1 \text { Xor 0x1B If False RC[11]= } \\
\operatorname{Rc}[10]<<1\end{array}$ & $0 \times 62$ \\
\hline 12 & $\begin{array}{l}\mathrm{RC}[11] \text { And } 0 \times 80 !=0 \text { ? If True } \mathrm{Rc}[12]=\mathrm{Rc}[11] \ll<1 \text { Xor 0x1B If False RC[12]= } \\
\operatorname{Rc}[11]<<1\end{array}$ & $0 x c 4$ \\
\hline 13 & $\begin{array}{l}\text { RC[12] And 0x80 != } 0 \text { ? If True Rc[13] }=\operatorname{Rc}[12] \ll<1 \text { Xor 0x1B If False RC[13]= } \\
\operatorname{Rc}[12] \ll<1\end{array}$ & $0 \times 92$ \\
\hline 14 & $\begin{array}{l}\mathrm{RC}[13] \text { And } 0 \mathrm{x} 80 !=0 \text { ? If True } \mathrm{Rc}[14]=\mathrm{Rc}[13] \ll<1 \text { Xor 0x1B If False RC[14]= } \\
\operatorname{Rc}[13]<<1\end{array}$ & $0 \times 3 e$ \\
\hline 15 & $\begin{array}{l}\mathrm{RC}[14] \text { And } 0 x 80 !=0 \text { ? If True } \mathrm{Rc}[15]=\operatorname{Rc}[14] \ll<1 \text { Xor 0x1B If False RC[15]= } \\
\operatorname{Rc}[14] \ll<1\end{array}$ & $0 \times 7 c$ \\
\hline 16 & $\begin{array}{l}\mathrm{RC}[15] \text { And } 0 \times 80 !=0 \text { ? If True } \mathrm{Rc}[16]=\mathrm{Rc}[15] \ll<1 \text { Xor 0x1B If False RC[16]= } \\
\mathrm{Rc}[15] \ll<1\end{array}$ & $0 x f 8$ \\
\hline
\end{tabular}


Analysis of the key scheduling process in Noekeon's algorithm can be described as follows:

Key Scheduling :

Input Key : 12345000000000000

Key(Bit)

00110001;00110010;00110011;00110100;

00110101;00110000;00110000;00110000;

00110000;00110000;00110000;00110000;

00110000;00110000;00110000;00110000;

A0 : 00110001;00110010;00110011;00110100

$=825373492$

A1 : 00110101;00110000;00110000;00110000

$=892350512$

A2 : 00110000;00110000;00110000;00110000

$=808464432$

A3 : 00110000;00110000;00110000;00110000

$=808464432$

Round (0)Scheduling Key :

$\mathrm{A} 0=\mathrm{A} 0$ Xor $\mathrm{RC}(0)=825373620$

Theta Inv Process : A0 : 825373620

A1 : 892350512

A2 : 808464432

A3 : $808464432 \mathrm{~T}=\mathrm{A} 0$ Xor A2

$=16909188$

$\mathrm{A} 0=\mathrm{A} 0$ Xor $\mathrm{RC}(16)=2504158901 \mathrm{DK}(0)=$

$\mathrm{A} 0=2504158901$

$\mathrm{DK}(1)=\mathrm{A} 1=334622109 \mathrm{DK}(2)=\mathrm{A} 2=$

$3718075253 \mathrm{DK}(3)=\mathrm{A} 3=205715165$

Theta Inv Process :

A0 : 2504158901

A1 : 334622109

A2 : 3718075253

A3 : $205715165 \mathrm{~T}=\mathrm{A} 0$ Xor A2

$=1222589888$

Temp $=\mathrm{T}<<8$ Xor $\mathrm{T}>>8=527769461 \mathrm{~A} 1=\mathrm{T}$

Xor Temp Xor A1 = 1146867496 A3 $=$ T Xor

Temp Xor A3 $=1541985384 \mathrm{~T}=\mathrm{A} 1$ Xor A3 = 531831616

Temp $=\mathrm{T}<<8$ Xor $\mathrm{T}>>8=4077187844 \mathrm{~A} 0=\mathrm{T}$

Xor Temp Xor A0 $=2046134001$ A2 $=$ T Xor

Temp Xor A2 $=824884017$
Temp $=\mathrm{T}<<8$ Xor $\mathrm{T}>>8=2248312322 \mathrm{~A} 1=\mathrm{T}$

Xor Temp Xor A1 = 2989536694 A3 = T Xor

Temp Xor A3 $=3073422774 \mathrm{~T}=\mathrm{A} 1$ Xor A3 = 83886080

Temp $=\mathrm{T}<<8$ Xor $\mathrm{T}>>8=327685$

$\mathrm{A} 0=\mathrm{T}$ Xor Temp Xor A0 $=876032945 \mathrm{~A} 2=\mathrm{T}$

Xor Temp Xor A $2=892678197 \mathrm{~A} 1=\mathrm{A} 1<<1=$ 1684106093

$\mathrm{A} 2=\mathrm{A} 2<<5=2795898534 \mathrm{~A} 3=\mathrm{A} 3<<2=$

3703756506

Gamma Process :

A0 : 876032945

A1 : 1684106093

A2 : 2795898534

A3 : 3703756506

$\mathrm{A} 1=\mathrm{A} 1$ Xor $((\operatorname{Not} \mathrm{A} 3)$ And $(\operatorname{Not} \mathrm{A} 2))=$ 1702380140

$\mathrm{A} 0=\mathrm{A} 0$ Xor $(\mathrm{A} 2$ And $\mathrm{A} 1)=269955477 \mathrm{~A} 0=\mathrm{A} 3$

$=3703756506$

$\mathrm{A} 3=\mathrm{A} 0=269955477$

A2 $=$ A2 Xor A3 Xor A 1 Xor A0 $=252420997$

$\mathrm{A} 1=\mathrm{A} 1$ Xor $(\operatorname{Not} \mathrm{A} 3$ And Not A2) $=$

2241334790

$\mathrm{A} 0=\mathrm{A} 0$ Xor $(\mathrm{A} 2$ And $\mathrm{A} 1)=3653948638 \mathrm{~A} 1=$

A1 $\gg 1=1120667395$

$\mathrm{A} 2=\mathrm{A} 2 \gg 5=678976796 \mathrm{~A} 3=\mathrm{A} 3>2=$

1141230693

$\mathrm{I}(0)=\mathrm{A} 0=2046134001 \mathrm{I}(1)=\mathrm{A} 1=1146867496$

$\mathrm{I}(2)=\mathrm{A} 2=824884017 \mathrm{I}(3)=\mathrm{A} 3=1541985384$

Based on the analysis of key scheduling that has been done, the key is obtained for the encryption and decryption process as follows:

Input key : 12345 Encryption key :

$\mathrm{I}(0)=\mathrm{A} 0=2046134001 \mathrm{I}(1)=\mathrm{A} 1=1146867496$

$\mathrm{I}(2)=\mathrm{A} 2=824884017 \mathrm{I}(3)=\mathrm{A} 3=1541985384$

Decryption key :

$\mathrm{DK}(0)=\mathrm{A} 0=2504158901 \mathrm{DK}(1)=\mathrm{A} 1=$ $334622109 \mathrm{DK}(2)=\mathrm{A} 2=3718075253 \mathrm{DK}(3)=$ A3 $=205715165$

\subsection{Encryption Process Analysis}

Encryption analysis in Noekeon's algorithm uses input blocks of 32 bits in length. Here is the description of the operation of the encryption process in Noekeon's algorithm:

1. Input Plaintext from the user.

2. They are padding the plaintext if the mod length of 16 is not equal to 0 .

3. Form A0-A3 input blocks that are 32 bits or four characters long, respectively. 
4. Perform an encryption loop of 16 rounds(i) which can be described as follows.

1. Perform xor A0 operations with RC(i).

2. Perform theta operations against $\mathrm{A} 0-\mathrm{A} 3$ using $\mathrm{K}(0)-\mathrm{K}(3)$.

3. Perform rotate_left operations A $1<<1$

4. Perform operations rotate_left A2 $<<5$

5. Perform operations rotate_left A3 $<<2$

6. Perform gamma operations against A0-A3.

7. Perform rotate_right operation A1 $>1$

8. Perform rotate_right operation A2 $>5$

9. Perform operations rotate_right $\mathrm{A} 3>>2$

10. After the next round of operations, perform xor operations $\mathrm{A} 0$ and $\mathrm{RC}(16)$.

11. Perform theta operations against $\mathrm{A} 0-\mathrm{A} 3$ using $\mathrm{K}(0)-\mathrm{K}(3)$.

12. Break down blocks A0-A3 into bytes of ciphertext characters.

Analysis of the encryption process in Noekeon's algorithm can be described as follows.

Plaintext : "zizi encryption 12" Input Block

Formation :

A $0: 1701735282$

Input

Block

(0)

A1 : 1768977257

01100101;01101110;01101011;01110010

A2 : 544893306

Input

Block

A3 : 1763717426

01101001;01110000;01110011;01101001

Round (0) Encryption :

$\begin{array}{lcc}\text { Input } & \text { Block } & (2) \\ \text { 00100000;01111010;01101001;01111010 }\end{array}$

$\mathrm{A} 0=\mathrm{A} 0$ Xor $\mathrm{RC}(0)=1701735410$

Theta Process : A0 : 1701735410

Input

Block

(3)

A1 : 1768977257

01101001;00100000;00110001;00110010

A2 : 544893306

End (4) BLok encryption:

A3 : 1763717426 
$\mathrm{K}(0): 2046134001$

$\mathrm{K}(1): 1146867496$

$\mathrm{K}(2): 824884017$

$\mathrm{K}(3): 1541985384$

$\mathrm{T}=\mathrm{A} 0$ Xor A2

$=1158939272$

Temp $=\mathrm{T}<<8$ Xor $\mathrm{T}>>8=2621938759$

$\mathrm{A} 1=\mathrm{T}$ Xor Temp Xor A1 $=2955144614$

A $3=$ T Xor Temp Xor A3 $=2960371709$

$\mathrm{A} 0=\mathrm{A} 0$ Key $\operatorname{Xor}(0)=479980803$

$\mathrm{A} 1=\mathrm{A} 1 \mathrm{Key} \operatorname{Xor}(1)=4101513870$

A2 $=$ A2 Key Xor $(2)=290509387$

A3 : 2926440023

$\mathrm{A} 1=\mathrm{A} 1$ Xor ((Not A3) And (Not A2))

3923792957

$\mathrm{A} 0=\mathrm{A} 0$ Xor $(\mathrm{A} 2$ And $\mathrm{A} 1)=865995842$

$\mathrm{A} 0=\mathrm{A} 3=2926440023$

$\mathrm{A} 3=\mathrm{A} 0=865995842$

$\mathrm{A} 2=\mathrm{A} 2$ Xor A 3 Xor A1 Xor A0 $=2864567542$

Round operations are performed in 16 rounds. After the round is complete then the operation is continued as follows.

$\mathrm{A} 0=\mathrm{A} 0$ Xor $\mathrm{RC}(16)=2594189566$

Theta Process :

A0 : 2594189566

A1 : 347116736

A2 : 563119268

A3 : 4101676072

$\mathrm{K}(0): 2046134001$

$\mathrm{K}(1): 1146867496$

A2 Xor Key $(2)=280641429$

$\mathrm{A} 3=\mathrm{A} 3 \mathrm{Xor} \operatorname{Key}(3)=2125305365 \mathrm{~T}=\mathrm{A} 1 \mathrm{Xor}$

A $3=4286124968$

Temp $=\mathrm{T}<<8$ Xor $\mathrm{T}>>8=3521958380 \mathrm{~A} 0=\mathrm{T}$

Xor Temp Xor A0 $=3451941963$ A2 $=$ T Xor

Temp Xor A2 = 1043332561

After the process is complete, the ciphertext can be obtained by breaking block A0-A3 into bytes of characters that can be seen as follows:

Output(0) : 205

Output(1) : 192

Output(2) : 116

Output(3) : 75

Output(4) : 129

Output(5) : 212

\subsection{Decryption Process Analysis}

The decryption process in Noekeon's algorithm uses input blocks of 32 bits in length. Here is the description of the operation of the
$\mathrm{A} 3=\mathrm{A} 3 \mathrm{Xor} \operatorname{Key}(3)=3952835477$

$\mathrm{T}=\mathrm{A} 1$ Xor $\mathrm{A} 3=534993179$

Temp $=\mathrm{T}<<8$ Xor $\mathrm{T}>>8=4165400646$

$\mathrm{A} 0=\mathrm{T}$ Xor Temp Xor A0 $=4215163998$

$\mathrm{A} 2=\mathrm{T}$ Xor Temp Xor A2 $=4143280918$

$\mathrm{A} 1=\mathrm{A} 1<<1=3908060445$

$\mathrm{A} 2=\mathrm{A} 2<<5=3735970526$

$\mathrm{A} 3=\mathrm{A} 3<<2=2926440023$

Gamma Process :

A0 : 4215163998

A1 : 3908060445

A2 : 3735970526

$\mathrm{A} 1=\mathrm{A} 1$ Xor $(\operatorname{Not} \mathrm{A} 3$ And Not A2) $=$

2912974644

$\mathrm{A} 0=\mathrm{A} 0$ Xor $(\mathrm{A} 2$ And A1 $)=114145891$

$\mathrm{A} 1=\mathrm{A} 1>>1=1456487322$

$\mathrm{A} 2=\mathrm{A} 2>>5=3042307751$

$\mathrm{A} 3=\mathrm{A} 3>>2=2363982608$

encryption process in Noekeon's algorithm:

1. Input ciphertext from the user.

$\mathrm{K}(2): 824884017$

$\mathrm{K}(3): 1541985384 \mathrm{~T}=\mathrm{A} 0$ Xor A2

$=3140531290$

Temp $=\mathrm{T}<<8$ Xor $\mathrm{T}>>8=1779395087 \mathrm{~A} 1=$ $\mathrm{T}$ Xor Temp Xor A1 $=3314502293 \mathrm{~A} 3=\mathrm{T}$

Xor Temp Xor A3 = 625295997 A0 = A0 Key Xor $(0)=3814045199$

$\mathrm{A} 1=\mathrm{A} 1 \mathrm{Xor} \operatorname{Key}(1)=2178188733$

A2

Output(6) : 133

Output(7) : 189

Output $(8): 62$

Output(9) : 47

Output(10) : 253

Output(11) : 209

Output(12): 126

Output(13) : 173

Output(14) : 150

Output(15) : 21

Then obtained the results of encryption as

follows:

Plaintext : "Zizi encryption 12" Chiperteks :

${ }^{2} O s Y \Psi a$ aáy_êÛ $\square 1$ Š_(_[X_ä1 ${ }^{1} r \& v^{\prime \prime} 1 / 2\{$

2. Form $\mathrm{A} 0-\mathrm{A} 3$ input blocks that are 32 bits or 4 characters long, respectively.

3. Decryption rounds of 16 rounds(i) are performed from rounds $16-1$ (inverted) which can be described below.

4. Perform theta operations against A0-A3 using $\mathrm{K}(0)-\mathrm{K}(3)$. 
5. Perform xor A0 operations with RC(i).

6. Perform rotate_left operations A1 $<<1$

7. Perform operations rotate_left $\mathrm{A} 2<<5$

8. Perform operations rotate_left $\mathrm{A} 3<<2$

9. Perform gamma operations against A0- A3.

10. Perform rotate_right operation $\mathrm{A} 1>>$

11. Perform rotate_right operation $\mathrm{A} 2>>$

12. Perform operations rotate_right $\mathrm{A} 3>>$

13. Perform theta operations against $\mathrm{A} 0-\mathrm{A} 3$ using $\mathrm{K}(0)-\mathrm{K}(3)$.

14. Breaks block A0-A3 into bytes of ciphertext characters.

Analysis of the decryption process in Noekeon's algorithm can be described as follows:chipertext:

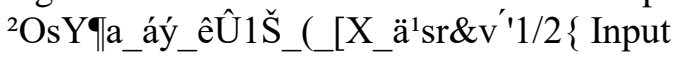
Block Formation :

$\begin{array}{lcc}\text { Input } & \text { Block } & (0) \\ 11001101 ; 11000000 ; 01110100 ; 01001011 \\ \text { Input } & \text { Block } & (1) \\ 10000001 ; 11010100 ; 10000101 ; 10111101 \\ \text { Input } & \text { Block } & (2) \\ \text { 00111110;00101111;11111101; } 11010001 \\ \text { Input } & \text { Block } & (3) \\ \text { 01111110;10101101;10010110;00010101 }\end{array}$

End (4) BLok

A0 : 3451941963

A1 : 2178188733

A2 : 1043332561

A3 : 2125305365

Round (0) Decryption :

Theta Process :

A0 : 3451941963

A1 : 2178188733

A2 : 1043332561

A3 : 2125305365

$\mathrm{K}(0): 2504158901$

$\mathrm{K}(1): 334622109$

$\mathrm{K}(2): 3718075253$

$\mathrm{K}(3): 205715165 \mathrm{~T}=\mathrm{A} 0$ Xor A2

$=4092561818$

Temp $=\mathrm{T}<<8$ Xor $\mathrm{T}>>8=1970959738 \mathrm{~A} 1=\mathrm{T}$

Xor Temp Xor A1 = 121731421 A3 $=$ T Xor

Temp Xor A3 $=4164446965$ A0 = A0 Key

Xor $(0)=1484921598$

$\mathrm{A} 1=\mathrm{A} 1 \mathrm{Xor} \operatorname{Key}(1)=347116736$ A2 = A2 Key

$\operatorname{Xor}(2)=3820138148$ A3 = A3 Xor Key $(3)=$

$4101676072 \mathrm{~T}=\mathrm{A} 1$ Xor A3 $=3771336936$

Temp $=\mathrm{T}<<8$ Xor $\mathrm{T}>>8=585638632$

$\mathrm{A} 0=\mathrm{T}$ Xor Temp Xor A $0=2594189566 \mathrm{~A} 2=$

$\mathrm{T}$ Xor Temp Xor A2 $=563119268$ A $0=$ A0 Xor
$\mathrm{RC}(0)=2594189318$

$\mathrm{A} 1=\mathrm{A} 1<<1=694233472 \mathrm{~A} 2=\mathrm{A} 2<<5=$ 839947396 A $3=$ A $3<<2=3521802403$

Gamma Process :

A0 : 2594189318

A1 : 694233472

A2 : 839947396

A3 : 3521802403

$\mathrm{A} 1=\mathrm{A} 1$ Xor $((\operatorname{Not} \mathrm{A} 3)$ And $(\operatorname{Not} \mathrm{A} 2))=$

627319512

$\mathrm{A} 0=\mathrm{A} 0$ Xor $(\mathrm{A} 2$ And $\mathrm{A} 1)=3131060358 \mathrm{~A} 0=$

$\mathrm{A} 3=3521802403$

$\mathrm{A} 3=\mathrm{A} 0=3131060358$

$\mathrm{A} 2=\mathrm{A} 2$ Xor A3 Xor A1 Xor A0 $=2084501113$

$\mathrm{A} 1=\mathrm{A} 1 \mathrm{Xor}(\operatorname{Not} \mathrm{A} 3$ And Not A2 $)=606415832$

$\mathrm{A} 0=\mathrm{A} 0$ Xor $(\mathrm{A} 2$ And $\mathrm{A} 1)=4123939579$

$\mathrm{A} 1=\mathrm{A} 1 \gg 1=303207916 \mathrm{~A} 2=\mathrm{A} 2 \gg>5=$

3420583859 A $3=\mathrm{A} 3>>2=2930248737$

Round operations are performed in 16 rounds. After the round is complete then the operation is continued as follows.

Theta Process :

A0 : 4215163998

A1 : 4101513870

A2 : 4143280918

A3 : 3952835477

$\mathrm{K}(0): 2504158901$

$\mathrm{K}(1): 334622109$

$\mathrm{K}(2): 3718075253$

$\mathrm{K}(3): 205715165 \mathrm{~T}=\mathrm{A} 0$ Xor A2

$=231423816$

Temp $=\mathrm{T}<<8$ Xor $\mathrm{T}>>8=2201125682 \mathrm{~A} 1=\mathrm{T}$

Xor Temp Xor A1 $=2055315188$ A3 $=$ T Xor

Temp Xor A3 = 1700972527 A0 = A0 Xor

$\operatorname{Kunci}(0)=1853629163$ A1 = A1 Xor Kunci $(1)=$ 1768977257 A2 $=$ A2 Xor Kunci $(2)=728244323$ A $3=$ A 3 Xor Kunci $(3)=1763717426 \mathrm{~T}=$ A 1 Xor $\mathrm{A} 3=5259867$

Temp $=\mathrm{T}<<8$ Xor $\mathrm{T}>>8=188877634 \mathrm{~A} 0=\mathrm{T}$ Xor Temp Xor A0 $=1701735410 \mathrm{~A} 2=\mathrm{T}$ Xor Temp Xor A2 $=544893306$ A0 $=$ A0 Xor RC $(0)$ $=1701735282$ 
After the process is complete, the plaintext can be obtained by splitting block A0-A3 into byte characters that can be seen as follows: Output $(0): 101$

Output(1) : 110

Output(2) : 107

Output(3) : 114

Output(4) : 105

Output(5) : 112

Output(6) : 115

Output(7) : 105

Output(8): 32

\author{
Output(9) : 122 \\ Output(10) : 105 \\ Output(11): 122 \\ Output(12) : 105 \\ Output(13) : 32 \\ Output(14) : 49 \\ Output(15): 50 \\ Then obtained the results of decryption as \\ follows: \\ Plaintext : "zizi encryption 12"
}

\section{CONCLUSION}

With the completion of this research which includes the process of encryption and decryption of messages using the Noekeon algorithm, it can be concluded that Noekeon's algorithm can be applied in encryption and decryption of text messages by dividing the characters contained in the text into blocks Noekeon blocks so that it can be processed into noekeon rotation operations into ciphertext or plaintext. The process of designing and developing a text message encoding application using Noekeon's algorithm begins with analyzing Noekeon's algorithm both in the process and its provisions. The Next Stage is to create a neocon interface and process modules consisting of key initialization, key generation, encryption, and decryption.

\section{REFERENCES}

[1] N. M. D. Oktafiansyah, F. Agus, and S. Maharani, "Penerapan Kriptografi Dengan Algoritma Data Encryption Standart Pada Text Hasil Konversi Dari Citra," Semin. Nas. Ilmu Komput. dan Teknol. Inf., vol. 1, no. 1, pp. 85-89, 2016.

[2] Y. Wiharto and A. Irawan, "Enkripsi Data Menggunakan Advanced Encryption Standart 256," Kilat, vol. 7, no. 2, pp. 91-99, 2018, doi: 10.33322/kilat.v7i2.352.

[3] S. Wardoyo and R. Fahrizal, "Aplikasi Teknik Enkripsi Dan Dekripsi File Dengan Algoritma Blowfish Pada Perangkat Mobile Berbasis Android," Setrum Sist. Kendali-Tenaga-elektronikatelekomunikasi-komputer, vol. 3, no. 1, p. 43, 2016, doi: 10.36055/setrum.v3i1.497.

[4] A. Saputra and A. Widyanto, "Enkripsi Dan Dekripsi File Dengan Algoritma Blowfish," J. Sist. Inf. Dan Teknol. Inf., vol. 4, no. 1, pp. 22-30, 2015.

[5] M. I. Assegaf, R. Destias, N. Sitaresmi, and Y. Wiharto, "Implementasi Enkripsi-Dekripsi dengan Algoritma RC2 Menggunakan Java," J. Media Inform. Budidarma, vol. 4, pp. 898-903, 2020, doi: 10.30865/mib.v4i4.2256.

[6] E. L. Hakim, Khairil, and F. H. Utami, "Aplikasi Enkripsi Dan Deskripsi Data Menggunakan Algoritma Rc4 Dengan Menggunakan Bahasa Pemrograman Php,” J. Media Infotama, vol. 10, no. 1, pp. 1-7, 2014.

[7] D. Adhar, "Implementasi Algoritma Des (Data Encryption Standard) Pada Enkripsi Dan Deskripsi Sms Berbasis Android," J. Tek. Inform. Kaputama, vol. 3, no. 2, pp. 53-60, 2019, [Online]. Available: https://jurnal.kaputama.ac.id/index.php/JTIK/article/view/185.

[8] A. H. Lubis, "ENKRIPSI DATA DENGAN ALGORITMA KRIPTROGRAFI NOEKEON," CESS(JournalOfComputerEngineering,System AndScience), vol. 2, no. 1, pp. 97-101, 2017.

[9] C. Kurniawan, "Algoritma Kriptografi Noekon," [Online]. Available: http://ilmusisteminfo.com/upload/file_pdf/Kriptografi dan Algoritma 1567687693.pdf.

[10] I. Utomo, W. Mulyono, W. S. Sari, D. R. Ignatius, M. Setiadi, and C. A. Sari, "M ODIFIKASI E NKRIPSI G AMBAR M ENGGUNAKAN 64- BIT K UNCI P ADA A LGORITMA D ATA E NCRYPTION S TANDARD ( DES ),” Din. Rekayasa, vol. 12, no. 2, 2018. 